\title{
La polyploïdisation des mégacaryocytes résulte d'une mitose incomplète
}

Les mégacaryocytes de mammifères ont la propriété unique d'être des cellules polyploïdes ayant un contenu en ADN de $2^{\mathrm{x}} \mathrm{N}$ avec un seul noyau multilobé. La ploïdie du mégacaryocyte varie de $2 \mathrm{~N}$ à $128 \mathrm{~N}$ avec une ploïdie modale de $16 \mathrm{~N}$ chez l'homme. Ce phénomène survient au cours de la différenciation terminale simultanément avec la synthèse des principales protéines plaquettaires et l'individualisation des organites caractéristiques de la lignée mégacaryocytaire/plaquettaire (granules $\alpha$ et membranes de démarcation). La polyploïdisation des mégacaryocytes est un phénomène qui est survenu au cours de l'évolution et qui est propre aux mammifères. Par exemple, les précurseurs des thrombocytes chez l'oiseau sont des cellules diploïdes. Les raisons précises de ce phénomène dans la biologie du mégacaryocyte sont inconnues. Mais il est évident qu'il existe un lien étroit entre polyploïdisation et production plaquettaire, les plaquettes résultant de la fragmentation $\mathrm{du}$ cytoplasme $\mathrm{du}$ mégacaryocyte. La polyploïdisation du mégacaryocyte s'accompagne quasi simultanément au cours de la différenciation d'une augmentation de la taille du cytoplasme du mégacaryocyte et du développement des membranes de démarcation, qui vont ainsi permettre une amplification majeure du nombre de plaquettes produites par mégacaryocyte. La polyploïdisation du mégacaryocyte est-elle donc une manière "économique» pour la cellule de produire des plaquettes ou, de manière plus générale, correspond-elle à un changement majeur dans la biologie du mégacaryocyte modifiant les fonctions des plaquettes produites?

Initialement (dans les années 19701980) le mécanisme aboutissant à la polyploïdisation du mégacaryocyte a été appelé endomitose, car la plupart des auteurs pensaient qu'un début de mitose survenait mais sans mouvement de chromosomes ni rupture de l'enveloppe nucléaire. Des observations d'endomitoses avaient été faites en ultrastructure, mais la rareté des mégacaryocytes et des endomitoses dans la moelle expliquait le caractère préliminaire de ces descriptions. Plus récemment (1993-1996), l'étude de lignées continues humaines ou murines exprimant un phénotype mégacaryocytaire a conduit plusieurs auteurs à faire l'hypothèse que la polyploïdisation du mégacaryocyte correspondrait, en fait, à des successions de duplications de l'ADN (phase $\mathrm{S}$ ), interrompues par des temps de repos (G1/G2), sans entrée en mitose.

La découverte récente de la thrombopoïétine, qui permet d'obtenir un grand nombre de mégacaryocytes en culture, rend possible une analyse directe de la biologie du mégacaryocyte. Deux articles récents [1,2], et dont les résultats concordent, apportent des éléments de réponse intéressants quant aux mécanismes des endomitoses. Les deux groupes ont étudié, par des techniques d'immunomarquage, les endomitoses des mégacaryocytes murins ou humains cultivés in vitro en présence de thrombopoḯtine. Tous deux concluent que les endomitoses du mégacaryocyte sont réellement des mitoses mais incomplètes. Les phases initiales de la mitose sont tout à fait normales : il y a rupture de l'enveloppe nucléaire, individualisation des chromosomes, et constitution d'un fuseau mitotique. Dans les mégacaryocytes polyploïdes, ce fuseau est multipolaire. La mitose a lieu tout à fait normalement jusqu'à la métaphase, mais la fin de la mitose est anormale. Il ne s'agit pas, cependant, d'un blocage dans la transition métaphase/anaphase, un point de contrôle important de la mitose. En effet, les chromatides migrent vers les différents asters. Néanmoins, cette migration est limitée par la structure même du fuseau, qui est particulièrement court. L'équipe de Nagata interprète ces données comme reflétant une absence d'anaphase B (phase d'élongation des microtubules) [1]. La mitose se termine ensuite par la reconstitution d'une enveloppe nucléaire qui englobe toutes les chromatides situées à proximité, sans que l'on puisse individualiser de vraie télophase et sans cytokinèse (division du cytoplasme).

Les anomalies moléculaires responsables de ces modifications sont actuellement inconnues mais, contrairement aux données obtenues précédemment à partir de lignées cellulaires, elles ne sont pas dues à l'absence de cycline B1, de Cdc2 ou d'une activité kinase associée à la cycline B1 [2].

La caractérisation précise de ces anomalies moléculaires (anomalies de régulation de la mitose ou absence de certaines kinésines) reste très importante pour mieux comprendre non seulement la biologie du mégacaryocyte, mais également la régulation de la division cellulaire.

N.V.

N.D.

W.V.

1. Nagata Y, Muro Y, Todokoro K. Thrombopoietin-induced polyploidization of bone marrow megakaryocytes is due to unique regulatory mechanism in late mitosis. J Cell Biol 1997; 139: 449-57.

2. Vitrat N, Cohen-Solal K, Pique C, Le Couedic JP, Norol F, Larsen AK, Katz A, Vainchenker W, Debili N. Endomitosis of human megakaryocytes are due to abortive mitosis. Blood 1998; 91 : 3711-23.
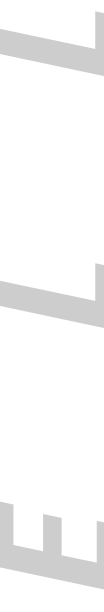

.

